\title{
Synchronization between Fractional-Order and Integer-Order Hyperchaotic Systems via Sliding Mode Controller
}

\author{
Yan-Ping Wu and Guo-Dong Wang \\ College of Science, Northwest A\&F University, Yangling, Shaanxi 712100, China \\ Correspondence should be addressed to Guo-Dong Wang; gd.wang@yahoo.cn
}

Received 16 September 2012; Accepted 19 January 2013

Academic Editor: Magdy A. Ezzat

Copyright ( 2013 Y.-P. Wu and G.-D. Wang. This is an open access article distributed under the Creative Commons Attribution License, which permits unrestricted use, distribution, and reproduction in any medium, provided the original work is properly cited.

The synchronization between fractional-order hyperchaotic systems and integer-order hyperchaotic systems via sliding mode controller is investigated. By designing an active sliding mode controller and choosing proper control parameters, the drive and response systems are synchronized. Synchronization between the fractional-order Chen chaotic system and the integer-order Chen chaotic system and between integer-order hyperchaotic Chen system and fractional-order hyperchaotic Rössler system is used to illustrate the effectiveness of the proposed synchronization approach. Numerical simulations coincide with the theoretical analysis.

\section{Introduction}

During the past decades, fractional calculus has become a powerful tool to describe the dynamics of complex systems such as power systems, mathematics, biology, medicine, secure communication, and chemical reactors [1-6]. Chaos synchronization has attracted lots of attention in a variety of research fields [7-13] over the last two decades, because it can be applied in vast areas of physics and engineering and secure communication $[14,15]$. Moreover, many theoretical analysis and numerical simulation results about the synchronization of chaotic systems are obtained. Wang et al. [16] deal with the finite-time chaos synchronization of the unified chaotic system with uncertain parameters. Chen and Liu [17] propose a simple linear state feedback controller to realize the stability control of a unified chaotic system. The problem of chaos synchronization between two different chaotic systems with fully unknown parameters is investigated in [18]. Moreover, Chen and his partners [19] investigate the chaos control of a class of fractional-order chaotic systems via sliding mode.

All of above articles mainly focus on integer-order chaotic systems or fractional-order chaotic systems. There is little information about the synchronization between fractionalorder chaotic systems and integer-order chaotic systems [20, 21]. The study of synchronization between fractional-order hyperchaotic systems and integer-order hyperchaotic systems is also limited.

Motivated by the above discussion, this paper investigates a sliding mode method for synchronization between a class of fractional-order hyperchaotic systems and integer-order hyperchaotic systems. And the integer-order hyperchaotic systems are regarded as response system in the proposed synchronous technique which is simple and theoretically rigorous.

\section{System Description and Problem Formulation}

Consider the following fractional-order hyperchaotic system as a drive system

$$
D^{q} x=A x+f(x)
$$

where $x(t) \in R^{4}$ denotes four-dimensional state vector. $A \in$ $R^{4 \times 4}$ represents the linear part of the system, and $f: R^{4} \rightarrow$ $R^{4}$ is the nonlinear part of the system.

And the response system can be described as

$$
D y=B y+g(y),
$$


where $y(t) \in R^{4}$ is four-dimensional state vector, $B \in R^{4 \times 4}$ and $g: R^{4} \rightarrow R^{4}$ imply the same roles as $A$ and $f$ in the drive system, respectively.

Remark 1. $A$ and $f(\cdot)$ in the drive system can be same as $B$ and $g(\cdot)$ in the response system, respectively.

One adds the controller $u(t) \in R^{4}$ into the response system, which is given by

$$
D y=B y+g(y)+u(t) .
$$

Define the synchronous errors as $e=y-x$. The aim is to choose a suitable controller $u(t) \in R^{4}$, so that the drive system and response system can achieve chaotic synchronization (i.e., $\lim _{t \rightarrow \infty}\|e\|=0$, where $\|\cdot\|$ is the Euclidean norm).

\section{Design of Sliding Mode Controller}

Let the controller $u(t)$ be

$$
u(t)=u_{1}(t)+u_{2}(t),
$$

where $u_{1}(t) \in R^{4}$ is a compensation controller and $u_{1}(t)=$ $D x-B(x)-g(x)$. Here, $x(t) \in R^{4}$ in the response system belongs to hyperchaotic fractional-order drive system. $u_{2}(t) \in R^{4}$ is a vector function, and it will be designed later.

From (4), the system (3) can be rewritten as

$$
D e=B e+g(y)-g(x)+u_{2}(t) .
$$

In accordance with the active control design procedure, the nonlinear part of the error dynamics is eliminated by the following choice of the input vector [22]

$$
u_{2}(t)=g(x)-g(y)+K w(t) .
$$

The error system (5) is rewritten as

$$
D e=B e+K w(t),
$$

where $K=\left[k_{1}, k_{2}, k_{3}, k_{4}\right]^{T}$ is a constant gain vector and $w(t) \in R$ is the control input which satisfies

$$
w(t)= \begin{cases}w^{+}(t) & s(e) \geq 0 \\ w^{-}(t) & s(e)<0\end{cases}
$$

To design a sliding mode controller, one has two steps. First, one constructs a sliding surface that represents a desired system dynamics. Next, one develops a switching control law such that a sliding mode exists on every point of the sliding surface, and any states outside the surface are driven to reach the surface in a finite time [23]. As a choice for the sliding surface, one has

$$
\begin{gathered}
s_{1}(t)=c_{1} e_{1}+c_{2} e_{2}, \\
s_{2}(t)=c_{2} e_{2}+c_{3} e_{3}, \\
s_{3}(t)=c_{4} e_{4}+c_{3} e_{3}, \\
s_{4}(t)=c_{4} e_{4},
\end{gathered}
$$

which can also be easily given by

$$
s(t)=C e, \quad \text { where } C=\left[\begin{array}{cccc}
c_{1} & c_{2} & 0 & 0 \\
0 & c_{2} & c_{3} & 0 \\
0 & 0 & c_{3} & c_{4} \\
0 & 0 & 0 & c_{4}
\end{array}\right] .
$$

In the sliding mode, the sliding surface and its derivative must satisfy

$$
s(t)=0, \quad \dot{s}(t)=0 .
$$

Consider

$$
\dot{s}(t)=D s=0 \Longrightarrow C D e+e=C(B e+K w(t))+e=0 .
$$

One can get that

$$
w(t)=-(C K)^{-1}(C B-I) e(t) .
$$

Replacing for $w(t)$ in (7) from $w(t)$ of (13), the error dynamics on the sliding surface are determined by the following relation:

$$
D e=\left(I-K(C K)^{-1} C\right) B e .
$$

To satisfy the sliding condition, the discontinuous reaching law is chosen as follows:

$$
D s=-p \operatorname{sign}(s)-r s,
$$

where

$$
\operatorname{sign}(s)= \begin{cases}+1, & s>0 \\ 0, & s=0 \\ -1, & s<0\end{cases}
$$

and $p>0, r>0$ are the gains of the controller.

In the sliding phase, it implies that $D s=\dot{s}(t)=0$. Considering (12) and (15), one gets

$$
w(t)=-(C K)^{-1}[C(r I+B) e+p \operatorname{sign}(s)] .
$$

Now, the total control law can be defined as follows:

$$
\begin{aligned}
u(t)= & D x-B x-g(y) \\
& -K(C K)^{-1}[C(r I+B) e+p \operatorname{sign}(s)] .
\end{aligned}
$$

Replacing $w(t)$ in (7) by (17), the error dynamics are determined by

$$
D e=\left[B-K(C K)^{-1} C(r I+B)\right] e-K(C K)^{-1} p \operatorname{sign}(s) .
$$

Theorem 2 (see [24]). The following system is as follows:

$$
D^{q} x=A x, \quad x(0)=x_{0},
$$

where $0<q \leq 1, x \in R^{n}$ and $A \in R^{n \times n}$. System (20) is asymptotically stable if $\left|\arg \left(\lambda_{i}\right)\right|>q \pi / 2$, where $\lambda_{i}$ are the eigenvalues of matrix A. Also, this system is stable if $\left|\arg \left(\lambda_{i}\right)\right| \geq$ $q \pi / 2$ and those critical eigenvalues that satisfy $\left|\arg \left(\lambda_{i}\right)\right|=$ $q \pi / 2$ have geometric multiplicity one. 
Theorem 3 (see [24]). Consider a system given by the following linear state space form with inner dimension $n$ as follows:

$$
\begin{gathered}
D^{q} x=A x+B u, \\
y=C x, \quad x(0)=x_{0},
\end{gathered}
$$

where $0<q \leq 1, x \in R^{n}, y \in R^{p}$, and $A \in R^{n \times n}$. Assuming that the triplet $(A, B, C)$ is minimal, then system (21) is stable if $|\arg (\operatorname{eig}(A))|>q \pi / 2$.

According to Theorem 2, the error dynamics on the sliding surface defined by (14) is asymptotically stable, as long as all eigenvalues of $\left[\left(I-K(C K)^{-1} C\right) B\right]$ satisfy the condition $\left|\arg \left(\lambda_{i}\right)\right|>\pi / 2$. In the sliding phase, as a linear fractionalorder system with bounded inputs $\left(-K(C K)^{-1} p\right.$ for $s>0$ and $K(C K)^{-1} p$ for $s>0$ ), the error system (19) is stable if $\left|\arg \left(\operatorname{eig}\left(\left[B-K(C K)^{-1} C(r I+B)\right]\right)\right)\right|>\pi / 2$. It can be shown that choosing appropriate $K, C$, and $r$ can make the error dynamics stable; hence, the synchronization is realized.

\section{Numerical Simulation}

This section presents two illustrative examples to verify and demonstrate the effectiveness of the proposed control scheme. Case 1 is the synchronization between the same structure hyperchaotic systems. Case 2 is the synchronization between the different structure hyperchaotic systems.

Case 1. Synchronization between fractional-order and integer-order hyperchaotic Chen systems. [25]

Consider Chen hyperchaotic system which is written as

$$
\begin{gathered}
\frac{d^{q_{1}} x_{1}}{d t^{q_{1}}}=a_{1}\left(x_{2}-x_{1}\right)+x_{4}, \\
\frac{d^{q_{2}} x_{2}}{d t^{q_{2}}}=\gamma x_{1}-x_{1} x_{3}+c_{1} x_{2}, \\
\frac{d^{q_{3}} x_{3}}{d t^{q_{3}}}=x_{1} x_{2}-b_{1} x_{3}, \\
\frac{d^{q_{4}} x_{4}}{d t^{q_{4}}}=x_{2} x_{3}+d_{1} x_{4} .
\end{gathered}
$$

When $q_{1}=q_{2}=q_{3}=q_{4}=1$, the system is integer-order system; otherwise we call the system (22) a fractional-order system.

Take the fractional-order system with fractional-order $q_{1}=q_{2}=q_{3}=q_{4}=0.95$ as a drive system, and the integer-order Chen hyperchaotic system as a response system with the following initial conditions: $\left[x_{1}, x_{2}, x_{3}, x_{4}\right]^{T}=$ $[0.1,0.5,-0.9,1]^{T}$ and $\left[y_{1}, y_{2}, y_{3}, y_{4}\right]^{T}=[0.1,0,0.9,0]^{T}$, and the system parameters are $\left(a_{1}, b_{1}, c_{1}, d_{1}, \gamma\right)=(35,3,28,7)$.

The controller parameters are chosen as $K=$ $\operatorname{diag}(-2,-8,-2,-2), C=[4,4,0,0 ; 0,4,4,0 ; 0,0,4,4$; $0,0,0,4], r=10$, and $p=2$. This selection of parameters results in eigenvalues $\left(\lambda_{1}, \lambda_{2}, \lambda_{3}, \lambda_{4}\right)=(-10,-10,-10,-10)$,

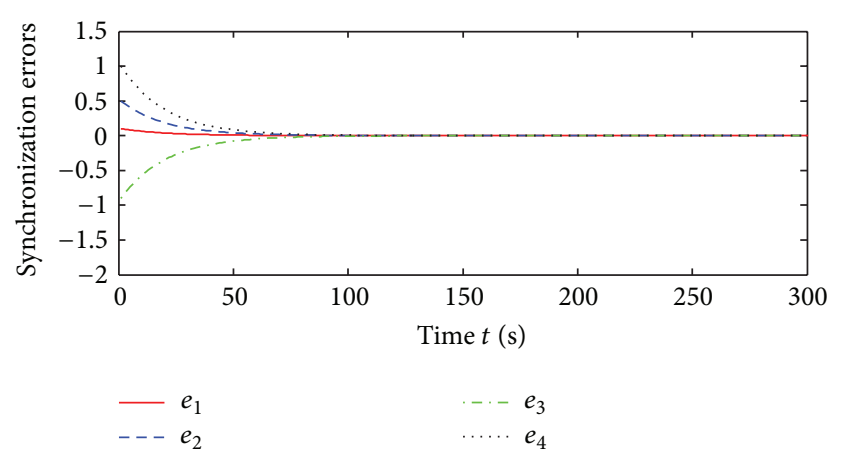

FIGURE 1: Synchronization errors between Chen systems.

which are located in the stable region. According to (18), the control inputs are taken as follows:

$$
\begin{aligned}
u_{1}= & \frac{d x_{1}}{d t}-a_{1}\left(x_{2}-x_{1}\right)-x_{4}+25 e_{1}-35 e_{2}-e_{4} \\
& -\frac{1}{2}\left(\operatorname{sign}\left(s_{1}\right)-\operatorname{sign}\left(s_{2}\right)+\operatorname{sign}\left(s_{3}\right)-\operatorname{sign}\left(s_{4}\right)\right), \\
u_{2}= & \frac{d x_{2}}{d t}-\gamma x_{1}-c_{1} x_{2}+y_{1} y_{3}-38 e_{2}-7 e_{1} \\
& +\frac{1}{2}\left(\operatorname{sign}\left(s_{3}\right)-\operatorname{sign}\left(s_{2}\right)-\operatorname{sign}\left(s_{4}\right)\right), \\
u_{3}= & \frac{d x_{3}}{d t}+b_{1} x_{3}-y_{1} y_{2}-7 e_{3}+\frac{1}{2}\left(\operatorname{sign}\left(s_{4}\right)-\operatorname{sign}\left(s_{3}\right)\right), \\
u_{4}= & \frac{d x_{4}}{d t}-d_{1} x_{4}-y_{2} y_{3}-\frac{21}{2} e_{4}-\frac{1}{2} \operatorname{sign}\left(s_{4}\right) .
\end{aligned}
$$

The simulation results are given in Figure 1. As we can see, the errors converge to zero which implies that synchronization between the two systems is realized.

Case 2. Synchronization between integer-order hyperchaotic Chen system and fractional-order hyperchaotic Rössler system. [26]

Consider hyperchaotic Rössler system which is written as

$$
\begin{gathered}
\frac{d^{q_{1}} x_{1}}{d t^{q_{1}}}=-x_{2}-x_{3}, \\
\frac{d^{q_{2}} x_{2}}{d t^{q_{2}}}=x_{1}+a_{2} x_{2}+x_{4}, \\
\frac{d^{q_{3}} x_{3}}{d t^{q_{3}}}=b_{2}+x_{1} x_{3}, \\
\frac{d^{q_{3}} x_{4}}{d t^{q_{3}}}=-c_{2} x_{3}+d_{2} x_{4} .
\end{gathered}
$$

Similarly, take the fractional-order Rössler hyperchaotic system with fractional-order $q_{1}=q_{2}=q_{3}=q_{4}=$ 0.95 as a drive system, and take the integer-order Chen 


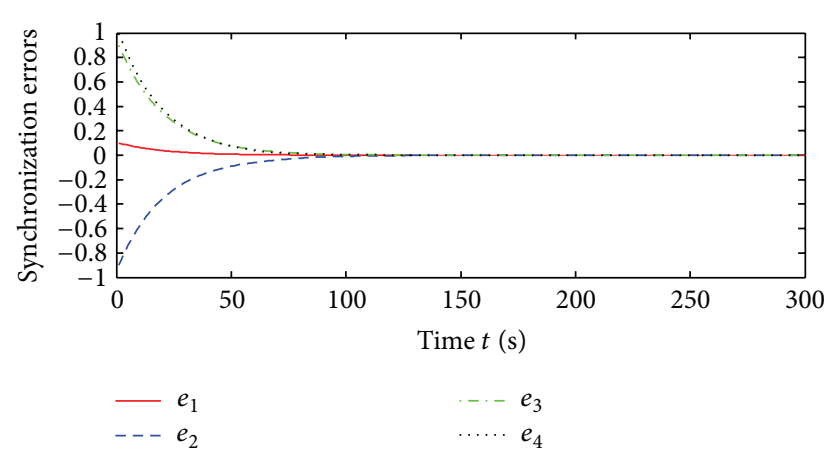

FIGURE 2: Synchronization between integer-order Chen system and fractional-order Rössler system.

hyperchaotic system as a response system with the following initial conditions: $\left[x_{1}, x_{2}, x_{3}, x_{4}\right]^{T}=[-0.1,-0.9,0.9,1]^{T}$ and $\left[y_{1}, y_{2}, y_{3}, y_{4}\right]^{T}=[-0.9,0.1,0.9,1.9]^{T}$, and the system parameters are $\left(a_{2}, b_{2}, c_{2}, d_{2}\right)=(0.25,3,0.5,0.05)$.

We choose the design parameters in the simulations as $K=\operatorname{diag}(-3,-5,-6,-3), C=[3,3,0,0 ; 0,3,3,0 ; 0,0,3,3$; $0,0,0,3], r=5$, and $p=0.2$. This selection of parameters results in eigenvalues, $\left(\lambda_{1}, \lambda_{2}, \lambda_{3}, \lambda_{4}\right)=(-5,-5,-5,-5)$, which are located in the stable region. According to (19), we can yield the response system easily, that is,

$$
\begin{aligned}
u_{1}= & \frac{d x_{1}}{d t}-a_{1}\left(x_{2}-x_{1}\right)-x_{4}+30 e_{1}-35 e_{2}-e_{4} \\
& -\frac{1}{15}\left(\operatorname{sign}\left(s_{1}\right)-\operatorname{sign}\left(s_{2}\right)+\operatorname{sign}\left(s_{3}\right)-\operatorname{sign}\left(s_{4}\right)\right), \\
u_{2}= & \frac{d x_{2}}{d t}-\gamma x_{1}-c_{1} x_{2}+y_{1} y_{3}-7 e_{1}-33 e_{2} \\
& +\frac{1}{15}\left(\operatorname{sign}\left(s_{3}\right)-\operatorname{sign}\left(s_{2}\right)-\operatorname{sign}\left(s_{4}\right)\right), \\
u_{3}= & \frac{d x_{3}}{d t}+b_{1} x_{3}-y_{1} y_{2}-2 e_{3}+\frac{1}{15}\left(\operatorname{sign}\left(s_{4}\right)-\operatorname{sign}\left(s_{3}\right)\right), \\
u_{4}= & \frac{d x_{4}}{d t}-d_{1} x_{4}-y_{2} y_{3}-\frac{11}{2} e_{4}-\frac{1}{15} \operatorname{sign}\left(s_{4}\right) .
\end{aligned}
$$

The synchronization errors are shown in Figure 2, which show that the proposed method is succeeded in synchronizing the two different structure systems.

\section{Conclusion}

In this paper, the problem of synchronization between fractional-order hyperchaotic systems and integer-order hyperchaotic systems is investigated. The integer-order hyperchaotic system is regarded as the response system. A sliding mode controller is designed to synchronize two systems with different orders successfully. It is rigorously proven that the proposed synchronization approach can be achieved between two different order hyperchaotic systems.
Some numerical simulations are presented to show the applicability and feasibility of the proposed scheme.

\section{Acknowledgments}

This wok was supported by the "111" Project from the Ministry of Education of People's Republic of China and the State Administration of Foreign Experts Affairs of People's Republic of China (B12007) and the National Science and Technology Supporting Plan from the Ministry of Science and Technology of People's Republic of China (2011BAD29B08).

\section{References}

[1] A. M. Harb and N. Abdel-Jabbar, "Controlling Hopf bifurcation and chaos in a small power system," Chaos, Solitons \& Fractals, vol. 18, no. 5, pp. 1055-1063, 2003.

[2] X. Su, "Boundary value problem for a coupled system of nonlinear fractional differential equations," Applied Mathematics Letters, vol. 22, no. 1, pp. 64-69, 2009.

[3] J. Ma, Y. Jia, M. Yi, J. Tang, and Y. F. Xia, "Suppression of spiral wave and turbulence by using amplitude restriction of variable in a local square area," Chaos, Solitons \& Fractals, vol. 41, no. 3, pp. 1331-1339, 2009.

[4] W. X. Wang, L. Huang, Y. C. Lai, and G. Chen, "Onset of synchronization in weighted scale-free networks," Chaos, vol. 19, no. 1, Article ID 013134, 2009.

[5] Z. Wei and Q. Yang, "Controlling the diffusionless Lorenz equations with periodic parametric perturbation," Computers \& Mathematics with Applications, vol. 58, no. 10, pp. 1979-1987, 2009.

[6] Y. Liu and Q. Yang, "Dynamics of a new Lorenz-like chaotic system," Nonlinear Analysis: Real World Applications, vol. 11, no. 4, pp. 2563-2572, 2010.

[7] D.-Y. Chen, W.-L. Zhao, X.-Y. Ma, and R.-F. Zhang, "Control and synchronization of chaos in RCL-shunted Josephson junction with noise disturbance using only one controller term," Abstract and Applied Analysis, vol. 2012, Article ID 378457, 14 pages, 2012.

[8] A. S. Hegazi and A. E. Matouk, "Dynamical behaviors and synchronization in the fractional order hyperchaotic Chen system," Applied Mathematics Letters, vol. 24, no. 11, pp. 19381944, 2011.

[9] D. Y. Chen, Z. T. Sun, X. Y. Ma, and L. Chen, "Circuit implementation and model of a new multi-scroll chaotic system," International Journal of Circuit Theory and Applications. In press.

[10] J. Zhang, K. Zhang, J. Feng, and M. Small, "Rhythmic dynamics and synchronization via dimensionality reduction: application to human gait," PLoS Computational Biology, vol. 6, no. 12, Article ID e1001033, 2010.

[11] D. Y. Chen, C. Wu, C. F. Liu, X.-Y. Ma, Y.-J. You, and R.-F. Zhang, "Synchronization and circuit simulation of a new double-wing chaos," Nonlinear Dynamics, vol. 67, no. 2, pp. 1481-1504, 2012.

[12] D.-Y. Chen, L. Shi, H.-T. Chen, and X.-Y. Ma, "Analysis and control of a hyperchaotic system with only one nonlinear term," Nonlinear Dynamics, vol. 67, no. 3, pp. 1745-1752, 2012.

[13] D. Chen, R. Zhang, X. Ma, and S. Liu, "Chaotic synchronization and anti-synchronization for a novel class of multiple chaotic systems via a sliding mode control scheme," Nonlinear Dynamics, vol. 69, no. 1-2, pp. 35-55, 2012. 
[14] D.-Y. Chen, W.-L. Zhao, X.-Y. Ma, and R.-F. Zhang, "Nochattering sliding mode control chaos in Hindmarsh-Rose neurons with uncertain parameters," Computers \& Mathematics with Applications, vol. 61, no. 10, pp. 3161-3171, 2011.

[15] L. Pan, W. Zhou, L. Zhou, and K. Sun, "Chaos synchronization between two different fractional-order hyperchaotic systems," Communications in Nonlinear Science and Numerical Simulation, vol. 16, no. 6, pp. 2628-2640, 2011.

[16] H. Wang, Z. Z. Han, Q. Y. Xie, and W. Zhang, "Finitetime chaos synchronization of unified chaotic system with uncertain parameters," Communications in Nonlinear Science and Numerical Simulation, vol. 14, no. 5, pp. 2239-2247, 2009.

[17] X. Chen and C. Liu, "Passive control on a unified chaotic system," Nonlinear Analysis: Real World Applications, vol. 11, no. 2, pp. 683-687, 2010.

[18] M. P. Aghababa, S. Khanmohammadi, and G. Alizadeh, "Finitetime synchronization of two different chaotic systems with unknown parameters via sliding mode technique," Applied Mathematical Modelling, vol. 35, no. 6, pp. 3080-3091, 2011.

[19] D.-Y. Chen, Y.-X. Liu, X.-Y. Ma, and R.-F. Zhang, "Control of a class of fractional-order chaotic systems via sliding mode," Nonlinear Dynamics, vol. 67, no. 1, pp. 893-901, 2012.

[20] Z. Ping, C. Yuan-Ming, and K. Fei, "Synchronization between fractional-order chaotic systems and integer orders chaotic systems (fractional-order chaotic systems)," Chinese Physics $B$, vol. 19, no. 9, Article ID 090503, 2010.

[21] D. Y. Chen, R. Zhang, J. C. Sprott, H. T. Chen, and X. Y. Ma, "Synchronization between integer-order chaotic systems and a class of fractional-order chaotic systems via sliding mode control," Chaos, vol. 22, no. 2, Article ID 023130, 2012.

[22] M. S. Tavazoei and M. Haeri, "Synchronization of chaotic fractional-order systems via active sliding mode controller," Physica A, vol. 387, no. 1, pp. 57-70, 2008.

[23] S. Dadras and H. R. Momeni, "Control of a fractional-order economical system via sliding mode," Physica A, vol. 389, no. 12, pp. 2434-2442, 2010.

[24] D. Matignon, "Stability results for fractional differential equations with applications to control processing," in Proceedings of the IMACS-IEEE/SMC Multiconference on Computational Engineering in Systems and Applications, vol. 2, pp. 963-968, Lille, France, 1996.

[25] C. Li and G. Chen, "Chaos in the fractional order Chen system and its control," Chaos, Solitons \& Fractals, vol. 22, no. 3, pp. 549-554, 2004.

[26] K. Moaddy, I. Hashim, and S. Momani, "Non-standard finite difference schemes for solving fractional-order Rössler chaotic and hyperchaotic systems," Computers \& Mathematics with Applications, vol. 62, no. 3, pp. 1068-1074, 2011. 


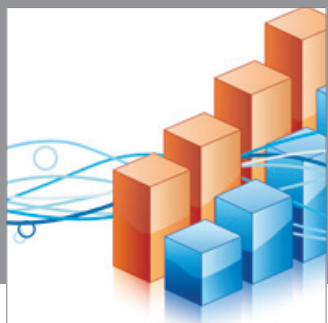

Advances in

Operations Research

mansans

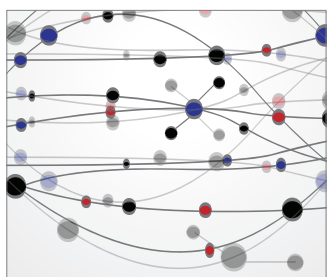

The Scientific World Journal
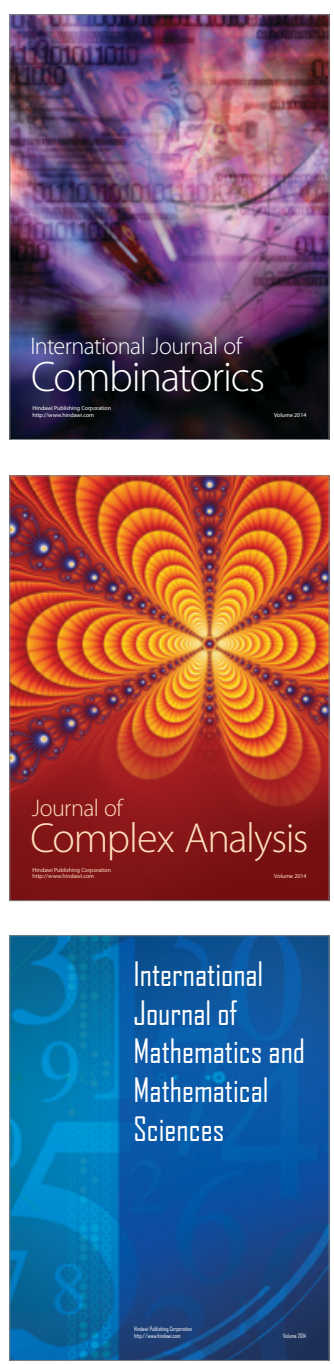
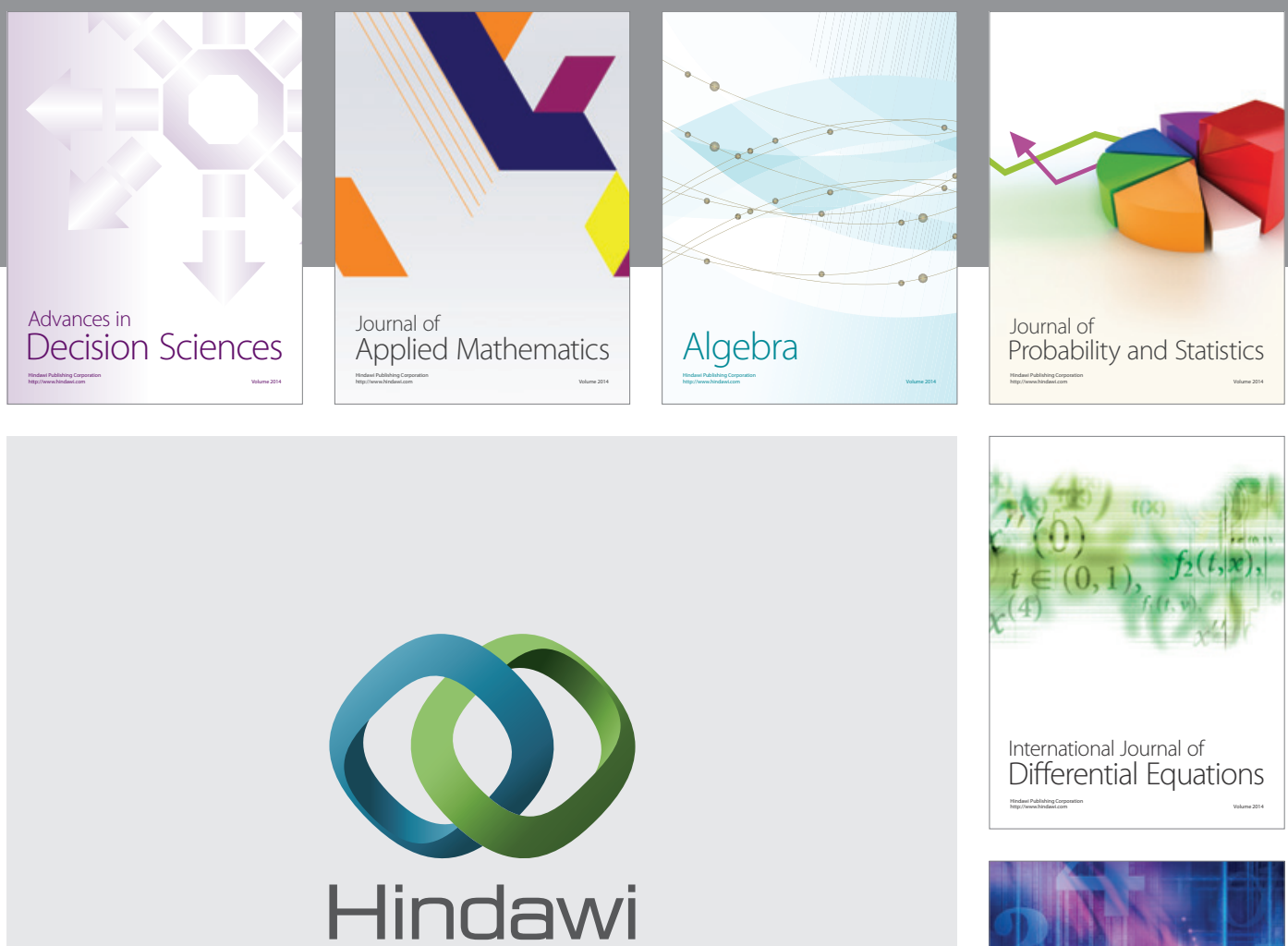

Submit your manuscripts at http://www.hindawi.com
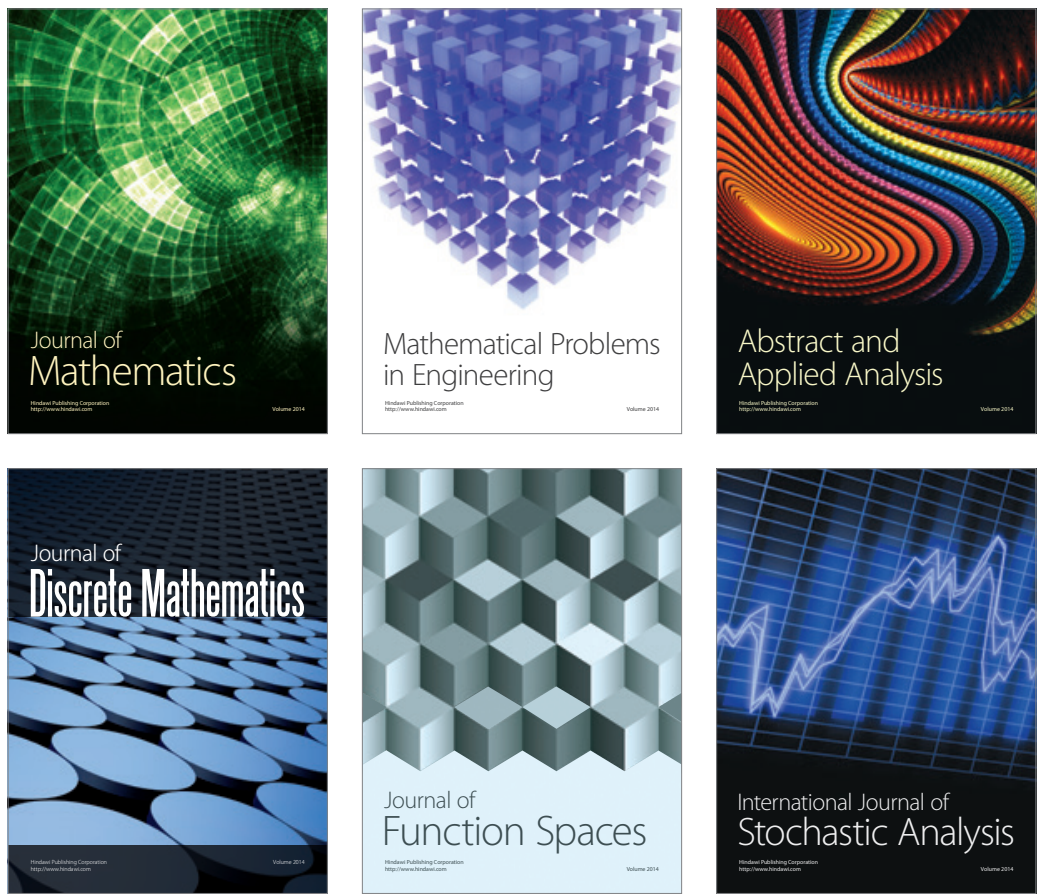

Journal of

Function Spaces

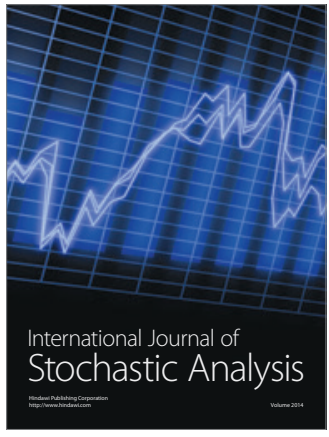

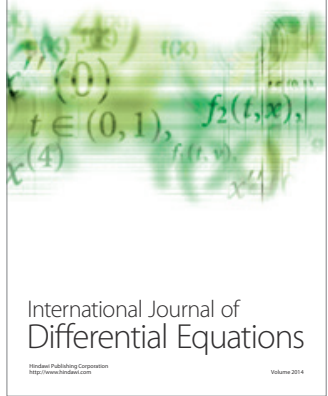
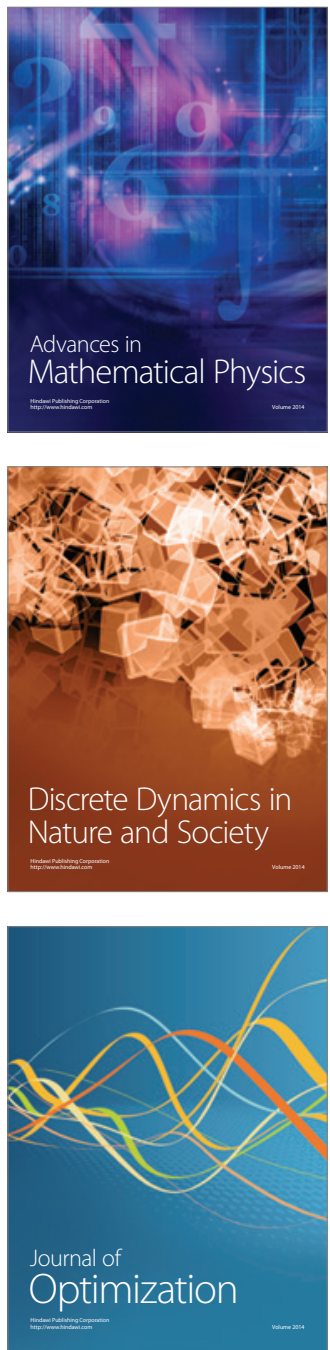